coloproctology 2021 · 43:251-252

https://doi.org/10.1007/s00053-021-00545-1

Angenommen: 3. Mai 2021

Online publiziert: 11. Juni 2021

(c) Springer Medizin Verlag $\mathrm{GmbH}$, ein Teil von Springer Nature 2021

Liebe Leserinnen und Leser,

die Entwicklung in der interdisziplinären Zusammenarbeit sowohl national als auch international ist unübersehbar. In allen Fachdisziplinen der Medizin bilden sich fachübergreifende Cluster. Es werden interdisziplinäre Zentren gegründet. Wie erfolgreich diese interdisziplinäre Zusammenarbeit sein kann, zeigt die aktuelle Entwicklung und Erforschung von Corona-Impfstoffen, bei der in Rekordzeit bestehende molekularbiologische Technologien zur Entwicklung eines Impfstoffes führten, wobei das Potenzial dieser neuen Technologien noch gar nicht abzuschätzen ist.

\section{》) Der Beckenboden stellt ein besonderes Organsystem für die interdisziplinäre Zusammenarbeit dar}

Gerade auch der Beckenboden stellt ein besonderes Organsystem für die interdisziplinäre Zusammenarbeit in multiprofessionellen Teams dar. Schon in den traditionellen Fächern laufen hier Urologie, Gynäkologie und die Proktologie vereinigt in einem komplexen funktionellen Organsystem zusammen. Insbesondere die Arbeiten aus den 1990er und 2000er Jahren von Peter Petros und Ulf Ulmsten veränderten die Blickweise in der Therapie von der rein anatomischen Perspektive hin zu einem funktionellen System, sodass heute neben der eigentlichen Behandlung der Primärerkrankung der Erhalt der Funktionalität des Beckenbodens im Vordergrund steht.

\title{
Markus Grebe
}

Standort Friedrichstadt, Städtisches Klinikum Dresden, Dresden, Deutschland

\section{Interdisziplinäre Behandlung gynäkologischer Erkrankungen im proktologischen Kontext}

Die Urogynäkologie und rekonstruktive Beckenbodenchirurgie sind neben der Tumorchirurgie ein wichtiges Feld der interdisziplinären Zusammenarbeit am Beckenboden. Nach eigenen Erfahrungen benötigen ca. $10 \%$ der gynäkologischen Deszensuspatienten eine gleichzeitige proktologische Therapie, z.B. im Rahmen eines gleichzeitig vorliegenden rektoanalen Vorfalls, einer Intussuszeption oder funktionellen Störungen, wie chronischen Defäkationsobstruktionen.

In der Tumorchirurgie ermöglichen Operationen in embryonalen Kompartimenten sowie der Einsatz neoadjuvanter Therapieverfahren immer häufiger einen Organ- und Funktionserhalt. Ein weiteres Feld der interdisziplinären Zusammenarbeit im Bereich des Beckenbodens sind enterovaginale Fisteln, z. B. bei chronisch-entzündlichen Darmerkrankungen. Im internationalen Kontext in den weniger entwickelten Gebieten besitzt das große Thema der geburtshilflichen Verletzungen und rektovaginalen Fisteln nach protrahierten Geburtsverläufen hohe persönliche und gesellschaftliche Relevanz.

Vulva und Analkarzinome treten in den letzten Jahren immer häufiger bei jungen Frauen im Zusammenhang mit einer HPV-Infektion auf. Bereits 2006 wurde in den USA und Europa der HPVImpfstoffs Gardasil zugelassen, welcher sowohl gegen die häufigsten Zervixkarzinom-induzierende Virustypen HPV 16 oder 18 als auch gegen die typischen Vulva-Papilloma-Virustypen 5 und 11 wirksam ist. Im Jahr 2015 erfolgte dann die Zulassung des Impfstoffs Gardasil 9 in der EU, welcher sowohl gegen die klassischen Virustypen 6, 11, 16, 18 als auch gegen die selteneren, jedoch häufig auch im Bereich der Vulva anzutreffenden Virustypen $31,33,45,52$ und 58 wirksam ist. Jiayao Lei et al. publizierten im New England Journal of Medicine 2020 eine altersadjustierte Reduktion von $49 \%$ der Inzidenz für Gebärmutterhalskrebs bis zum 31. Geburtstag in Schweden [1].

Seit fast 15 Jahren impfen die niedergelassenen Gynäkologen Mädchen gegen HPV, und seit Kurzem werden auch die Jungen von Gynäkologen und Kinderärzten prophylaktisch zum Erreichen einer Herdenimmunität gegen HPV geimpft. Aufgrund der langen Inkubationszeit von der Infektion über die zervikalen als auch vulvären intraepithelialen Neoplasien hin zum invasiven Karzinom ist in der Inzidenz von prämalignen und malignen Erkrankungen der Vulva derzeit noch ein Anstieg der Inzidenzen zu beobachten. Da jüngere Frauen in der Regel regelmäßig die gynäkologische Vorsorge wahrnehmen, ist hier die Aufgabe des Gynäkologen, auch auf prämaligne Veränderungen des Anoderms zu achten und diese ggf. einer proktologischen Diagnostik zuzuführen. In der Zukunft sollte die Zahl der HPV-induzierten Anal-, Vulva und Zervixkarzinome also abnehmen.

\section{》) Ein zertifiziertes onkolo- gisches Zentrum sollte in der Behandlung von malignen Tumoren Standard sein}

Sollte es zu einem Vulva- oder Zervixkarzinom gekommen sein, ist der frühe Primärbefund meist gut operabel und von hervorragender Prognose. Gerade aber 
die selteneren fortgeschrittenen Karzinome und besonders die Rezidivtumoren lassen sich oft nur im interdisziplinären Kontext kurativ, häufig aber auch nur noch palliativ operieren. Die Exenteration ist hier eine, häufig palliative, aber mit hoher Patientenakzeptanz bei fortgeschrittenen Karzinomen und Rezidiven etablierte Methode [2].

Während in der Koloproktologie die neoadjuvante Strahlentherapie bereits fest etabliert ist, ist in der Gynäkologie die adjuvante Radiotherapie, die adjuvante Radiochemotherapie und in der Palliativsituation, insbesondere bei Blutungen, die palliative Strahlentherapie von Vulva, Vaginal- und Zervixkarzinomen derzeitiger Standard. Hierbei lassen sich poloproktologische Nebenwirkungen nicht immer vermeiden, auch wenn sich in der heutigen Strahlungsplanung die Organdosis der sensiblen Organsysteme, insbesondere Dünndarm, Blase und Rektum, bereits erheblich reduzieren ließ. Aufgrund der inzwischen hohen Heilungsraten und dem Langzeitüberleben stehen Langzeitnebenwirkungen der gynäkologischen Strahlentherapie immer mehr im Vordergrund. Die wichtigsten Nebenwirkungen einer Strahlentherapie bei gynäkologischen Tumoren sind die akute und chronische Proktitis mit Diarrhoen, teilweise Obstipation und analer Inkontinenz. Als besonders schwerwiegend ist die Strahlentherapieinduzierte Tumorneogenese, z.B. Induktion von Rektumkarzinomen sowie die Ausbildung von Fisteln, insbesondere bei der definitiven Radiochemound Brachytherapie fortgeschrittener Zervixkarzinome. Das Management von Spätkomplikationen und die ggf. operative Versorgung im vorbestrahlten Gebiet stellen eine interdisziplinäre Herausforderung an die Gynäkologie, Koloproktologie und Urologie dar, welche in einem interdisziplinären Zentrum am besten versorgt werden kann. Daher sollte ein zertifiziertes onkologisches Zentrum in der Behandlung von malignen Tumoren Standard sein.

\section{Nicht nur in der Onkologie ist Zusammenarbeit geboten}

Während schwere geburtstraumatische Verletzungen des Beckenbodens und Fisteln, insbesondere in Afrika, einen hohen Stellenwert besitzen, sind diese in Deutschland ein seltenes Ereignis. Jedoch ist das Beckenbodentrauma in der Geburtshilfe auch in unserer zunehmend alternden Bevölkerung ein wichtiges Thema. So muss man damit rechnen, dass bis zu $50 \%$ der weiblichen Bevölkerung im Laufe ihres Lebens eine Beckenbodensymptomatik entwickeln. Die eine Gruppe sind die Harn- und Stuhlinkontinenzen, die andere sind die große Gruppe der Deszensuserkrankungen, die in einem Beckenbodenzentrum behandelt werden sollten. Im Beckenbodenzentrum sind Urologen, Gynäkologen und Koloproktologen in Kombination mit Radiologie, Physiotherapie, Geriatrie und Neurologie die wesentlichen Behandlungspartner im Rahmen der Beckenboden-Senkungsund Inkontinenztherapie. Stuhlentleerungsstörungen bei rektoanaler Intersuszeption bzw. rektovaginale Entero-/ Rektozelen sollten im interdisziplinären Team behandelt werden. In den letzten Jahrzenten wandelte sich hier die Therapie von teilweise schwer verstümmelnden, verschließenden Operationen (z.B. Kolpokleisis) hin zur komplexen Rekonstruktion des Beckenbodens mit Funktionserhalt, sowohl der Ausscheidungs- und Haltefunktion des urogenitalen und rektoanalen Komplexes als auch der Sexualfunktion bis ins hohe Alter. Hier sei auf die Arbeiten von Baessler et al. mit dem validierten deutschen Beckenbodenfragebogen hingewiesen, welcher uns in der täglichen Routine die Möglichkeit zur interdisziplinären Evaluation der Beckenbodensymptomatik gibt. Um der Patientin unnötige Folgeeingriffe zu ersparen, sollte in interdisziplinären Beckenbodenkonferenzen gemeinsam ein optimiertes Therapieschema festgelegt werden. So ist es in unserem Beckenbodenzentrum üblich, in der Beckenbodenkonferenz auch Kombinationseingriffe, z.B. die Rekto- pexie, wenn indiziert, in Kombination mit der Sakrokolpopexie minimal-invasiv durchzuführen.

\section{Dr. M. Grebe}

\section{Korrespondenzadresse}

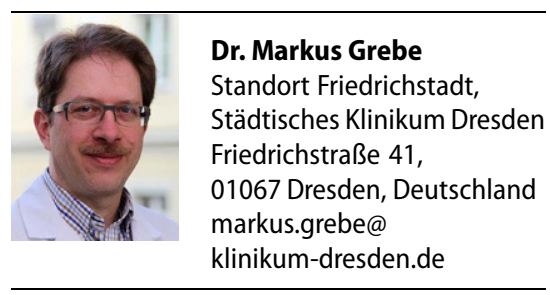

Interessenkonflikt. M. Grebe gibt an, dass kein Interessenkonflikt besteht.

\section{Literatur}

1. Lei J, Ploner A, Elfström KM, Wang J, Roth A, Fang F, Sundström K, Dillner J, Sparén P (2020) HPV Vaccination and the risk of invasive cervical cancer. N Engl J Med 383(14):1340-1348. https://doi.org/ 10.1056/NEJMoa1917338

2. Rost M (2004) Exenterative Chirurgie gynäkologischer Malignome: Ergebnisse nach dorsale Blasenteilresektion mit Erhalt des natürlichen Harnabflussweges. Dissertation, LMU München. https://doi.org/10.5282/edoc.1899 